\title{
MEDICAL DEVICE DESIGN PRACTITIONER STRATEGIES FOR PROTOTYPE-CENTERED FRONT-END DESIGN STAKEHOLDER ENGAGEMENTS IN LOW-RESOURCE SETTINGS
}

\author{
Coulentianos, Marianna J.; Rodriguez-Calero, Ilka; Daly, Shanna R.; Burridge, Jocelyn; Sienko, \\ Kathleen $\mathbf{H}$. \\ University of Michigan
}

\begin{abstract}
Prototypes have the potential to provoke discussion and to encourage stakeholders to play an active role during design engagements in the front-end phases of a design process. However, detailed descriptions of stakeholder engagement strategies in front-end design are lacking. The aim of this research study was to understand how design practitioners prepare and manage stakeholders for engagements involving prototypes in the front-end phases of a medical device design process. Design practitioners at companies developing mechanical and electromechanical medical devices for use in low- and middle-income countries were interviewed following a semi-structured interview guide. Interview transcripts were analysed, and inductive codes were developed. The findings suggest that design practitioners manage the group composition of stakeholders, review the project and prototype(s) with stakeholders at the start of the engagement, and show the progress of prototypes to stakeholders over multiple engagements. These strategies shed light on the importance of handling interpersonal relationships during stakeholder engagement with prototypes.
\end{abstract}

Keywords: User centred design, Early design phases, Design methods, Stakeholder engagement, Medical device design

Contact:

Coulentianos, Marianna Joy

University of Michigan

Design Science

United States of America

mjcoul@umich.edu

Cite this article: Coulentianos, M.J., Rodriguez-Calero, I., Daly, S.R., Burridge, J., Sienko, K.H. (2019) 'Medical Device Design Practitioner Strategies for Prototype-Centered Front-End Design Stakeholder Engagements in Low-Resource Settings', in Proceedings of the 22nd International Conference on Engineering Design (ICED19), Delft, The Netherlands, 5-8 August 2019. DOI:10.1017/dsi.2019.101 


\section{INTRODUCTION}

Stakeholder engagement is encouraged during medical device development (Caldwell et al., 2011) and prototypes are often recommended during later stages of concept development to gather feedback from stakeholders (Yock et al., 2015). Best practices related to this intersection of stakeholders and prototypes have primarily focused on the "back-end" of design, when concepts are more final, for example, best practices in usability testing (Wiklund et al., 2015).

The "front-end" of design, which includes problem definition, requirements elicitation, and early concept generation (Khurana and Rosenthal, 1998), also offers many opportunities for stakeholder engagement with prototypes through methods such as interviews and focus groups (Anderson, 2009; DiCicco-Bloom and Crabtree, 2006; Kitzinger, 1995). For example, when eliciting requirements, a front-end design activity focused on defining design constraints, a team described using early stage prototypes during interviews with stakeholder to incite dialogue and gain understanding of the problem (Green et al., 2015). Maiden and Rugg (1996) noted that the use of scenarios and prototypes in requirements elicitation sheds light on "taken-for-granted issues" because they help stakeholders recall latent knowledge. Nuseibeh and Easterbrook (2000) recommend using prototypes during stakeholder engagements to provoke discussion, and Wohlin and Aurum (2006) state that the use of prototypes can encourage stakeholders to "play an active role" during a feedback session. Further, Sanders and Stappers (2014) recommend that designers use probes - cultural and inspirational artifacts - to provoke reactions from stakeholders, that designers make toolkits to empower stakeholders to codesign in the process, and that designers use prototypes to give form to a potential solution and test it with stakeholders.

Guidelines for stakeholder interactions across a design process emphasize best practices in the "leadin" of the engagement. For instance, building trust at the beginning of design interviews is crucial for increasing the quantity and quality of information stakeholders share (Jacob and Furgerson, 2012). In a usability setting, the moments at the start of the engagement, including the greeting and the briefing, have been discussed as paramount to ensuring a productive engagement session (Wiklund et al., 2015). Furthermore, guidelines for informing the number and composition of stakeholders engaged in a focus group setting facilitate elicitation of a diverse range of perspectives while keeping the conversation focused (Rabiee, 2004). Similarly, designers likely employ specific strategies when using prototypes during front-end design engagements with stakeholders. Prototypes are effective tools for supporting communication with stakeholders and for engaging with stakeholders throughout a design process to support product success (Lauff et al., 2017; Vinck et al., 2003). However, there is a lack of specificity in how prototypes are leveraged in the front-end during stakeholder interactions across design contexts, thus studies of the methods designers use for engaging stakeholders using prototypes during front-end design warrant further investigation.

Specific guidance for how to stage stakeholder engagements with prototypes may be especially important in global health contexts. In these contexts, methods of stakeholder engagement used in highincome country settings may not be appropriate (Dupas, 2014; Mangham et al., 2009) and the hierarchy between designer and stakeholders can be accentuated (Donaldson, 2009), thus the value of the information collected could depend heavily on the set up of the feedback session and how well it mitigates such factors. Further, stakeholders and designers often live on different continents, which can make interactions brief and communication harder (Chavan et al., 2009; Donaldson, 2009), so any opportunity to engage stakeholders should be leveraged as best as can be. Hence, our study leveraged interviews with medical device design practitioners within the global health domain to investigate their strategies to set the stage for stakeholder engagement with prototypes during front-end design work.

\section{METHODS}

\subsection{Research aims}

This study aimed to understand how design practitioners initiate stakeholder interactions with prototypes in the front-end phases of designs of medical devices intended for use in low-resource settings, guided by the following research question: How do design practitioners stage stakeholder engagements that leverage prototypes during front-end design to elicit high quality feedback? 


\subsection{Participants}

Twelve practitioners from nine companies developing mechanical and electromechanical medical devices for use in low- and middle-income countries were included in the study. Potential participants were contacted by email, and if interested, they were asked to complete a background questionnaire that asked about their experiences using prototypes to engage stakeholders during the front-end design of a medical device for low-resource settings. Among the respondents, participants were selected for inclusion in the study if they stated sufficient experience engaging stakeholders with prototypes (answers: 'sometimes' and 'often').

Nine participants were working for companies based in the US, two for a company in Norway, and one for a company in India. Two participants worked for non-profit companies, four worked for private for-profit companies, one for a partnership company, one for a public for-profit company, and one for a sole proprietorship. Participants had a median of six years of experience (range: 0-30 years of experience, data missing for one participant) in design; two participants were medical doctors with experience in the development of medical devices; while they "developed" devices, they did not recognize this was "design work" and thus listed zero years of prior design experience. All participants held at least a bachelor's degree.

The study was approved by the University of Michigan Institutional Review Board (IRB). Participation was voluntary with written consent, and participants received $\$ 75$ for their participation.

\subsection{Data collection}

Semi-structured interviews were used to gather detailed information from participants (Roller and Lavrakas, 2015), in this case specific stories of design practitioners' prototype usage during stakeholder engagement in the front-end phases of design. Semi-structured interviews enable similar questions to be asked across participants while still allowing for additional questions to be asked in response to participant answers. Half of the interviews were conducted via video call over BlueJeans Video Conferencing $\odot$ the other half were conducted in person. On average, interviews lasted 93 minutes. The semi-structured interview protocol was developed through multiple rounds of iteration following pilot interviews. Established guidelines (Jacob and Furgerson, 2012) were followed for writing the interview protocol; specifically, developing open-ended interview questions based on the research questions and relevant literature, writing a script with additional prompts for each interview question, starting with easy and basic descriptive questions. The final version of the interview protocol had two phases. First the participant was asked to recall a project during which they had used prototypes to engage stakeholders in the design process and were asked about the circumstances of these interactions. Specifically, the interview explored what prototypes were used by the design practitioners and which stakeholders they engaged, how they engaged stakeholders during different activities of front-end design (problem identification, requirements elicitation, concept generation, concept selection), how they used parallel versus serial prototyping, and how they learned about the environment of use. In the second phase, the participant was asked to compare across projects. Interviews were audio-recorded.

\subsection{Data analysis}

Interview audio data were transcribed and de-identified. The transcripts were read multiple times to identify patterns for how prototypes were leveraged. Once preliminary patterns were established, NVivo software was used to identify specific instances of these patterns and a codebook was developed that described each of these patterns. The reliability of these codes was checked with a second rater. Finally, it was determined how many of the 12 participants described the strategy in the experiences they discussed during their interviews.

\section{RESULTS}

Three primary strategies emerged that represent how design practitioners prepare and manage the stakeholders for the engagement when leveraging prototypes, to mitigate potential hierarchical issues and gather quality feedback. The list of codes is presented in Table 1. 
Table 1. Definition of inductive strategies

\begin{tabular}{|c|c|c|}
\hline Strategy & Strategy Definition & $\begin{array}{c}\text { \# of } \\
\text { Participants }\end{array}$ \\
\hline $\begin{array}{c}\text { Strategy \#1 } \\
\text { Manage group } \\
\text { composition and size }\end{array}$ & $\begin{array}{c}\text { Design practitioners manage stakeholder group composition and } \\
\text { size to gather diverse perspectives and minimize bias due to } \\
\text { hierarchical relationships. }\end{array}$ & 5 \\
\hline $\begin{array}{c}\text { Strategy \#2 } \\
\text { Review the project } \\
\text { and prototype(s) }\end{array}$ & $\begin{array}{c}\text { At the start of the engagement, design practitioners summarize the } \\
\text { project, present the prototype(s), describe the role of the } \\
\text { stakeholder, and define expectations for the engagement and the } \\
\text { project. }\end{array}$ & 11 \\
\hline $\begin{array}{c}\text { Strategy \#3 } \\
\text { Show progress of } \\
\text { prototypes }\end{array}$ & $\begin{array}{c}\text { When stakeholders are engaged multiple times throughout the } \\
\text { design process, design practitioners show stakeholders updates } \\
\text { made to the prototype(s) to demonstrate the impact of } \\
\text { stakeholders' feedback on the project }\end{array}$ & 5 \\
\hline
\end{tabular}

\subsection{Strategy \#1: Manage group composition}

When planning for the engagement, participants described paying special attention to the stakeholders' group compositions when preparing focus groups that involved prototypes during the front-end phases of design. They described preferring homogeneous (i.e., minimal hierarchical differences among focus group members) and manageable group sizes.

Participants described approaches to achieve the "right" diversity of stakeholders, while making sure there were not too many people in the room during the engagement, to allow for more in-depth feedback:

"During a presentation, I want to get asked questions and want to respond to people, but I don't feel like you get the same in-depth feedback or the same in-depth concept generation that you do versus a one-on-one or even two people that are engaged, preferably from different points of view. (...) usually engaging stakeholders is kind of one stakeholder segment, and I wanted to get more diversity of stakeholders in a single room." (Participant F)

Participant $\mathrm{F}$ described the hard choice between gathering a diverse group of people representing multiple points of view versus holding in-depth one-on-one conversations with stakeholders. Participant $\mathrm{M}$ expressed frustrations with engagements involving too many stakeholders:

"I remember one meeting we had, where there were about 24 people in the room, and did not make much sense to me, so absolutely. Correctly, identify eight to ten people maximum, to really demonstrate to them the product, let them play with the product, answer the questions, identify what solutions they have." (Participant M)

Participants described managing group composition to lessen the impact of hierarchical relationships that sometimes prevented stakeholders from expressing themselves. They discussed how they mitigated the impact of hierarchical relationships by asking stakeholders to provide their feedback in written form and minimizing potential power differences among stakeholders present at the engagement:
"Yeah, I would say it's also about who else is there during the interaction because you have some countries that you work in, they are very hierarchical, so if you want to get feedback from certain stakeholders, you might have to do it separate from other ones because if for example my senior cardiologist is here, I won't answer any of the questions or correct him because he is a senior and he is the one with the knowledge. But in reality, that senior cardiologist hasn't practiced in 20 years. So, it would much more relevant to get the junior's feedback, so I need to make sure I get them away." (Participant $K$ )

This strategy aimed to minimize the likelihood of a dominant voice in the group and to establish a comfort level among all stakeholders participating in the engagement so that their knowledge, expertise, and feedback could be presented.

\subsection{Strategy \#2: review the project and prototype(s)}

At the beginning of an engagement involving one or more prototype(s), most participants discussed how they prepared stakeholders by reviewing the project and the prototype(s). Participants did so by 
giving a high-level overview of the project; presenting the prototypes and explaining their current form; defining the role of the stakeholder in the engagement; and defining expectations for the engagement and the project, as discussed by participant F:

"So, what I tended to do is introduce the problem, state why we were there, and then pull out the prototype, show some specific aspects that we are looking for feedback that were maybe missing in other interviews or other things we got were applicable to their specific discipline."

Participants described initiating the engagement by giving stakeholders a high-level overview of the project. In describing the prototypes, participants emphasized that its form was preliminary in nature. This strategy was particularly important because in the early phases of a design process, a prototype cannot stand on its own since it is preliminary and often has low fidelity elements.

"We could, ideally, choose to show any level of prototype to anyone, but we just put a caveat telling, 'This is a work in progress. Don't assume that this is the final product.'...” (Participant E)

Participants also discussed their strategies for making stakeholders feel like they could contribute to the design process by emphasizing the applicability of their expertise to the project. To do so, participants asked questions in ways that prompted wary stakeholders to provide criticism throughout the engagement. Participant $\mathrm{M}$ described how she phrased certain questions to encourage hesitant stakeholders to share feedback.

"It is the way you create the environment. Initially people don't talk, we think that we are so big experts, that (...) we don't need any such feedback, but then when you turn the table, and you tell them that their feedback makes the product for them, they really talk.(...) They are very humble, they never suggest anything, this is good. Then I will say, 'If you were to find out one mistake, or if you wanted one more thing, if you had an opportunity to change one more thing in this product, what would you like to do?"' (Participant M)

Finally, at the start of the engagement, participants managed stakeholder expectations on the topics of stakeholder participation and project evolution, as described by participant K. In this example, the prototype plays a central role in setting the expectations.

"One of them was about kind of stakeholder expectations and being able to find that right balance for how often to engagement and having stakeholders being very clear what their engagement means and how to kind of balance their expectations because as I was saying, it's helpful to bring them along in the process so they get to see what it kind of turns into but also sometimes stakeholders will not understand why you did not include something if you 're not able to explain it or show it through prototyping. (Participant $K$ )

\subsection{Strategy \#3: show progress of prototypes}

During the feedback session, some participants showed stakeholders how prototyping progress had been informed by the stakeholder's prior feedback to support stakeholders' understanding about design process, how prior feedback had informed iterations of design ideas, and contribute to relationship-building between designers and stakeholders.

Participant K describes how using this strategy created an understanding among the stakeholders about design processes and provided tangible examples of the design decisions involved in the development of the medical device:

"What makes an interaction easy is I think having (...) some rapport already. Having if it's a partner that we've been working with for a long time and I think also having them understand how we work, makes it a lot easier because sometimes when you work with new stakeholders and they don't understand the product development process, their expectations are not really aligned with really their own needs. (...) We try to have a design that's built, like we're building upon the stakeholders and having them for example engage throughout the process and being able to show how their input has influenced the next thing. (...) [It is] letting them be part of [the design] process and see the choices and understand why we're making different choices." (Participant $K$ )

Participant $M$ described how she maintained contact with various stakeholders of the project and showed them how their ideas had been integrated into the design. This encouraged stakeholders to continually contribute feedback, which helped the design team uncover critical requirements for the design: 


\begin{abstract}
"if we had not met with these stakeholders during the process, we would not have been able to make those very small, minor looking, most critical changes in the design. (...) As we were developing this product, we always remained in touch with the end users, the program managers, the policymakers, and other key stakeholders, and kept them up to date with what is happening. We sought their feedback and once we got the feedback we made changes into the design and we again went back to them, either face-to-face, or via communications to say: your idea has been incorporated. That's what we did." (Participant M)
\end{abstract}

Participant $\mathrm{G}$ further describes the importance of re-engaging stakeholders and showing the progress done on prototypes based on their feedback, a practice through which the stakeholders become part of the design process and that is perceived to yield feedback as important as usability testing.

"The other goal is we want (...) to be able to say the customer was very much a part of our design process, as any of the designers in-house were. (...) I'll say, 'Okay, we're going to start working on this stuff, and I'm going to come back in six months. Is that okay with you?' (...) We go back with models, and I'll get back and go to those same clinicians that I've observed, and say, 'Hey, because of what you lent to us through your time, six months ago when we were spending days here, these are some of the ideas we've come up with', prototypes. I'll just sit down with them in their laboratory, or in their clinical setting, and just get feedback on where we are. I've already done some usability, but I've gone from usability now to, 'Hey, I want to go to those original clinicians that I studied and see what they think about it."' (Participant G)

The practice of showing stakeholders the progression of prototypes enabled design practitioners in this scenario to build rapport with stakeholders, thus establishing a propitious long-lasting relationship between the designer and the stakeholder, which built trust: the stakeholders knew they were being heard and that their feedback was valued.

\title{
4 DISCUSSION
}

Three primary strategies emerged from the data with respect to preparing and managing stakeholder engagements that leverage prototypes during the front-end phases of a design process. Some participants emulated well-established best practices from qualitative research methods (Kitzinger, 1995) when selecting stakeholders - group size and the role composition - for a focus group, or found ways to mitigate the negative effects of overcrowded rooms or the presence of hierarchical relations among stakeholders during the engagement (strategy \#1). Similar to the trade-offs discussed by Kitzinger (1995), participants expressed a tension between speaking to homogeneous groups to capitalize on shared experiences versus exploring differences in perspectives within diverse groups.

When reviewing the project and prototype(s) (strategy \#2), participants discussed presenting the project and providing information about the prototype, which specifically included an explanation of the device's form factor. This strategy is similar to a strategy described by Wiklund et al. (2015) who recommend providing some basic information about the medical device through a written summary or video prior to conducting usability testing. An explanation of the form factor is of particular importance during front-end design engagements with stakeholders due to the low fidelity nature of prototypes. Low-fidelity prototypes have historically been heavily used in the field of software engineering and users compensate for bad aesthetics in lower fidelity prototypes during formative usability testing of software (Sauer and Sonderegger, 2009). However, prototype format is known to influence stakeholder feedback. Deininger et al. (2017) showed that prototype format (sketch, CAD model, cardboard mock-up, 3D print) affected the quality of actionable responses given by stakeholders. Hence, designers should consider potential effects on stakeholder feedback of the fidelity level of the particular form factor presented.

Participants also described taking time at the start of an engagement involving a prototype to make stakeholders feel like they were experts and had valuable information to share (strategy \#2). Indeed, stakeholders often feel apprehension (i.e., feel as if they cannot contribute anything of value) during an ethnographic interview (Spradley, 1979). Making the stakeholder feel less apprehensive may become more challenging with the introduction of the prototype, because the prototype could be perceived as solely within the domain expertise of the designer, which may in turn further distance the stakeholder. Deininger et al. (2017) showed that the questions posed by designers when engaging a stakeholder with a prototype can influence the quality of feedback elicited and discussed how the phrasing of the 
interview question accompanying the presentation of the prototype may serve to either empower or intimidate stakeholders.

Further, when reviewing the project and prototype(s) (strategy \#2), participants discussed the value of presenting background information and setting expectations, which are also best practices for stakeholder engagement during usability testing (Wiklund et al., 2015) because stakeholders who participate in design activities for the first time often do not know what to expect nor do they understand the purpose of the engagement (Spradley, 1979).

Traditional rapport-building strategies were discussed by participants throughout the interviews, such as being introduced by a trusted person or establishing rapport with the stakeholders prior to the engagement. Establishing rapport is an essential step to a good stakeholder engagement session and encourages stakeholders to share; however, typically there is little time to build rapport at the start of the engagement (DiCicco-Bloom and Crabtree, 2006). Using small talk and broad open-ended questions at the beginning of the interview and a usability test is recommended to make the interviewee comfortable (DiCicco-Bloom and Crabtree, 2006; Wiklund et al., 2015). Building rapport is especially important in a global health setting where there can be substantial cultural differences and power dynamics playing out between designer and stakeholder (Seyler et al., 2009). Participants mentioned leveraging prototypes to set expectations and build rapport with stakeholders, in order to elicit better feedback; specifically, they reviewed the project and prototypes with the stakeholders (strategy \#2) and showed stakeholders how their input impacted design changes (strategy \#3). Strategy \#2 is consistent with Chamorro-Premuzic et al. (2010), who find it critical that stakeholders evaluating a prototype must be familiar with the background and context of the device. Strategy \#3 is consistent with reported best practices of rapport building with stakeholders in agile methodology, where customers (who are also the users of the end-product) and designers are collocated for repeated opportunities of feedback gathering on various prototyped software releases with the same users (Fruhling and Vreede, 2006). In both strategy \#2 and \#3, design practitioners described the prototype as playing a central role.

Our findings demonstrate that established design approaches can be leveraged when preparing for and conducting engagements with stakeholders involving prototypes in the front-end of design. Additionally, design practitioners should devise supplementary strategies to support such engagements, such as explaining the form of the prototype and building rapport by showing stakeholders prototype progression. These strategies were reported to have increased the quality of feedback from stakeholders when potential hierarchies were at play, whether among stakeholders or among stakeholders and the designer.

Limitations of the study included a small sample size and focus on a single product domain. Future work will triangulate the self-reported practices with observational data during actual stakeholder engagements with prototypes. The findings can be leveraged by both design practitioners and design educators as scaffolding strategies to support novice designers stage stakeholder interactions with prototypes.

\section{CONCLUSION}

This preliminary study provides insights into how design practitioners within global health contexts structure stakeholder interactions with prototypes during the front-end design. Design practitioners discussed reviewing the project and prototype(s) with stakeholders at the start of the engagement, showing the progress of prototypes to stakeholders over multiple engagements, and managing the group composition of stakeholders in order to develop rapport as well as increase the likelihood of eliciting actionable design input.

\section{REFERENCES}

Anderson, K. (2009), "Ethnographic research: A key to strategy", Harvard Business Review, Vol.87 No. 3, p. 24. Caldwell, A., Young, A., Gomez-Marquez, J. and Olson, K.R. (2011), "Global Health Technology 2.0", IEEE Pulse, Vol. 2 No. 4, pp. 63-67.

Chamorro-Premuzic, T., Arteche, A., Bremmer, A.J., Greven, C. and Furnham, A. (2010), "Soft skills in higher education: importance and improvement ratings as a function of individual differences and academic performance", Educational Psychology, Vol. 30 No. 2, pp. 221-241.

Chavan, A.L., Gorney, D., Prabhu, B. and Arora, S. (2009), "The washing machine that ate my sari-mistakes in cross-cultural design", Interactions, Vol. 16 No. 1, pp. 26-31. 
Deininger, M., Daly, S., Sienko, K., Lee, J., Obed, S. and Kaufmann, E. E. (2017), "Does prototype format influence stakeholder design input?", 21st International Conference on Engineering Design (ICED 17) Vancouver, Canada, 21-25.08.2017. DS 87-4 Proceedings of the 21st International Conference on Engineering Design (ICED 17) Vol 4: Design Methods and Tools

DiCicco-Bloom, B. and Crabtree, B.F. (2006), "The qualitative research interview”, Medical Education, Vol. 40 No. 4, pp. 314-321.

Donaldson, K. (2009), “The Future of Design for Development: Three Questions”, Information Technologies and International Development, Vol. 5 No. 4, pp. 97-100.

Kroll, G., Carpena, F., Ghosh, I., Letouzé, E., Rosa, J. and Trivedi, P. (2014), "Revealing Demand for Pro-Poor Innovations" Revealing Demand for Pro-Poor Innovations. Georgetown University, March 7th 2014.

Fruhling, A. and Vreede, G.-J.D. (2006), "Field Experiences with eXtreme Programming: Developing an Emergency Response System”, Journal of Management Information Systems, Vol. 22 No. 4, pp. 39-68.

Green, J., Marnewick, A. and Pretorius, J. (2015), "Prototyping during the requirements elicitation process in the development of an underground unmanned aerial system", presented at the Pattern Recognition Association of South Africa and Robotics and Mechatronics International Conference (PRASA-RobMech), November 26, 2015, Port Elizabeth, Pattern Recognition Association of South Africa and Robotics and Mechatronics International Conference (PRASA-RobMech) pp. 60-65.

Jacob, S. and Furgerson, S. (2012), "Writing interview protocols and conducting interviews: Tips for students new to the field of qualitative research", The Qualitative Report, Vol. 17 No. 42, pp. 1-10.

Khurana, A. and Rosenthal, S.R. (1998), “Towards holistic 'front ends' in new product development”, Journal of Product Innovation Management, Vol. 15 No. 1, pp. 57-74.

Kitzinger, J. (1995), “Qualitative research: Introducing focus groups”, BMJ, Vol. 311 No. 7000, pp. 299-302.

Lauff, C., Kotys-Schwartz, D. and Rentschler, M.E. (2017), "What is a prototype?: Emergent roles of prototypes from empirical work in three diverse companies", presented at the ASME 2017 International Design Engineering Technical Conferences and Computers and Information in Engineering Conference, ASME, Cleveland, Ohio, USA, August 6-9, 2017, Volume 7: 29th International Conference on Design Theory and Methodology, p. V007T06A033.

Maiden, N.A.M. and Rugg, G. (1996), “ACRE: selecting methods for requirements acquisition”, Software Engineering Journal, Vol. 11 No. 3, pp. 183-192.

Mangham, L.J., Hanson, K. and McPake, B. (2009), "How to do (or not to do)... Designing a discrete choice experiment for application in a low-income country", Health Policy and Planning, Vol. 24 No. 2, pp.151158.

Nuseibeh, B. and Easterbrook, S. (2000), "Requirements engineering: A roadmap", Conference on the Future of Software Engineering, Limerick, Ireland, June 4-11, 2000, Proceedings of the Conference on the Future of Software Engineering, ACM, Vol. 1, pp. 35-46.

Rabiee, F. (2004), "Focus-group interview and data analysis", The Proceedings of the Nutrition Society, Vol. 63 No. 4, pp. 655-660.

Roller, M.R. and Lavrakas, P.J. (2015), Applied Qualitative Research Design: A Total Quality Framework Approach, Guilford Publications.

Sanders, E.B.-N. and Stappers, P.J. (2014), "Probes, toolkits and prototypes: three approaches to making in codesigning”, CoDesign, Vol. 10 No. 1, pp. 5-14.

Sauer, J. and Sonderegger, A. (2009), "The influence of prototype fidelity and aesthetics of design in usability tests: Effects on user behaviour, subjective evaluation and emotion”, Applied Ergonomics, Vol. 40 No. 4, pp. 670-677.

Seyler, D., Kang, K. and Place, M. (2009), Developing new products for emerging markets: a competency based approach, Theses, Rochester Institute of Technology

Spradley, J.P. (1979), The Ethnographic Interview, Holt, Rinehart and Winston.

Vinck, D., Blanco, E., Bovy, M., Laureillard, P., Lavoisy, O., Mer, S., Ravaille, N., et al. (2003), Everyday Engineering: An Ethnography of Design and Innovation, MIT Press.

Wiklund, M.E., Kendler, J., Strochlic, A.Y., Kendler, J. and Strochlic, A.Y. (2015), Usability Testing of Medical Devices, CRC Press, available at:https://doi.org/10.1201/b19082.

Wohlin, C. and Aurum, A. (2006), Criteria for Selecting Software Requirements to Create Product Value: An Industrial Empirical Study, in Biffl, S., Aurum, A., Boehm, B., Erdogmus, H. and Grünbacher, P. (Eds.), Value-Based Software Engineering, Springer Berlin Heidelberg, Berlin, Heidelberg, pp. 179-200.

Yock, P.G., Zenios, S., Makower, J., Brinton, T.J., Kumar, U.N. and Watkins, F.T.J. (2015), Biodesign: The Process of Innovating Medical Technologies, 2nd ed., Cambridge University Press, United Kingdom.

\section{ACKNOWLEDGMENTS}

We thank all participants of our study who took the time to have long in-depth conversations about their design process. This material is based upon work supported by the National Science Foundation under Grant No. 1745866 and Grant No. 2017248628. 